\title{
Rainmaker: A Tangible Work-Companion for the Personal Office Space
}

\author{
Sujay Shalawadi \\ sujay.shalawadi@uni-weimar.de \\ Mobile Media Group, Bauhaus \\ Universität \\ Weimar, Germany
}

\author{
Anas Alnayef \\ anas.alnayef@uni-weimar.de \\ Mobile Media Group, Bauhaus \\ Universität \\ Weimar, Germany
}

\author{
Niels van Berkel \\ nielsvanberkel@cs.aau.dk \\ Department of Computer Science, \\ Aalborg University \\ Aalborg, Denmark
}

\author{
Jesper Kjeldskov \\ jesper@cs.aau.dk \\ Department of Computer Science, \\ Aalborg University \\ Aalborg, Denmark
}

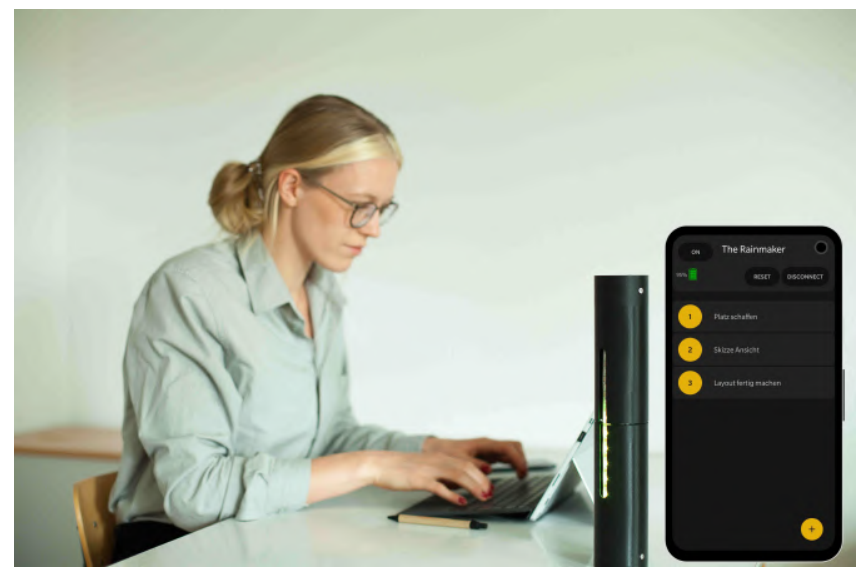

(a) User working with Rainmaker in the background
Florian Echtler

floech@cs.aau.dk

Department of Computer Science,

Aalborg University

Aalborg, Denmark

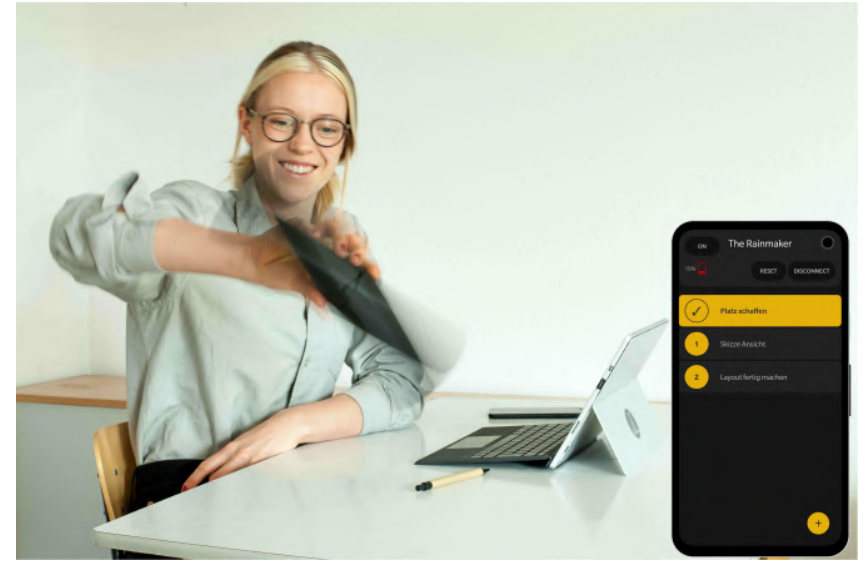

(b) Turning over Rainmaker to tick off a task

Figure 1: Two photos of the Rainmaker in use by a study participant.

\begin{abstract}
Routines are an important element of day-to-day work life, supporting people in structuring their day around required tasks. Effectively managing these routines is, however, experienced as challenging by many - an issue further amplified by the current work from home lockdown measures. In this paper we present Rainmaker, a tangible device to support people in their working life in the context of their own homes. We evaluate and iterate on our prototype through two qualitative studies, spanning respectively three days $(N=11)$ and 15 days $(N=2)$. Our results highlight the perceived advantages of the use of a primarily physical rather than digital tool for work support, allowing users to stay focused on their tasks and reflect on their work achievements. We present lessons for future work in
\end{abstract}

This work is licensed under a Creative Commons Attribution International 4.0 License.

MobileHCI '21, September 27-October 1, 2021, Toulouse \& Virtual, France (c) 2021 Copyright held by the owner/author(s)

ACM ISBN 978-1-4503-8328-8/21/09.

https://doi.org/10.1145/3447526.3472032 this area and publicly release the software and hardware used in the construction of Rainmaker.

\section{CCS CONCEPTS}

- Human-centered computing $\rightarrow$ User centered design.

\section{KEYWORDS}

Work from Home; Self Reflection; Multimodality; Reward; Work Routines

\section{ACM Reference Format:}

Sujay Shalawadi, Anas Alnayef, Niels van Berkel, Jesper Kjeldskov, and Florian Echtler. 2021. Rainmaker: A Tangible Work-Companion for the Personal Office Space. In Proceedings of the 23rd International Conference on Mobile Human-Computer Interaction (MobileHCI '21), September 27-October 1, 2021, Toulouse \& Virtual, France. ACM, New York, NY, USA, 13 pages. https://doi.org/10.1145/3447526.3472032

\section{INTRODUCTION}

The ability to develop routines is a critical skill for both productivity and well-being while working from home (WFH). Current 
global lockdowns as a result of the global Covid-19 pandemic have forced many to temporarily abandon their typical workspaces, with research indicating that WFH will remain a popular and common configuration beyond the current pandemic [3]. While current assessments of this work setup highlight numerous benefits, including some reports pointing to increased productivity [11], reduced infrastructure costs for office spaces, and the reduction of commuting time, thereby encouraging organisations to support WFH [13], several clear downsides of the current lockdown have also emerged. A recent survey among 1014 participants from countries most severely affected by the pandemic shows that the most common disadvantage of WFH is the lack of social contact, followed immediately by the lack of work-life balance and an inability to focus on work [32]. While people appreciate the flexibility of work timings in WFH, a negative impact on their job satisfaction can be seen due to lack of effective time boundaries between work and private life [4].

Time management during working hours is crucial in order to ensure subsequent leisure time. As the passage of time is perceived relatively rather than physically, task engagement plays a large role in this process (e.g., time passes by more slowly when completing a boring or challenging task) [12]. Time management can become challenging when creating new work routines, an issue highlighted by the wide range of available desktop and smartphone applications that aim to support effective time management techniques (e.g., Pomodoro, Get Things Done, Eisenhower matrix) and as extensively studied by the HCI community (see e.g., [1, 7, 24]). Although these applications provide people with the opportunity to experiment with structured and guided approaches to improve their task management skills and build towards a productive work routine, these applications often lack comprehensive support in relation to task visibility, reminders to refocus after interruptions, and a sense of progress. Additionally, the presence of these solutions within the digital working environment (desktop apps) or on potentially distracting personal devices (smartphone apps) may negatively affect their users' ability to switch between different contexts.

In this paper, we present the design and field evaluation of a prototype, named Rainmaker, designed to improve people's time management while WFH through multimodal interactions. The goal of our prototype is to introduce a tangible companion that enables people to develop their productivity and organisation of tasks in the context of WFH. Rainmaker, as shown in Figure 1, makes use of visual, auditory, and embodied interactions to support task organisation and execution for people during everyday work hours - supporting the process of having routines even in unfamiliar circumstances. We conducted two qualitative user studies to understand the effects of the prototype on productivity and efficient time management, as well as its ability to support positive work-life balance during the challenging times of physical isolation.

The subsequent sections of the paper are organised as follows: Section 2 describes existing works with multimodal interactions for representing time along with other prior work that has used tangible principles for supporting routines. Section 3 describes the salient features that fit our goal and the implementation of building a high fidelity prototype and an Android application. Section 4 presents the qualitative user study and the underlying research questions. Section 5 and 6 describe the procedure and participants of the two subsequent user studies along with the collected results.
Section 7 opens a discussion on our prototype and the use of tangible devices to support in WFH, while highlighting potential areas for future work. Finally, in Section 8 we conclude the paper.

\section{RELATED WORK}

Balancing activities in one's life in order to support well being is extensively researched and much has been written in the HCI community in relation to quantified self (QS) [29, 37]. Here, we explore existing works in the scope of quantified self, tangible rewards, self reflection, and physical interfaces for providing awareness and quantification between people and their work time. The latter can also be termed as quantified self at work (QSW) [27]. The works discussed below inspired the design of the Rainmaker.

\subsection{Physical Interfaces for the Quantified Self}

Apart from a multitude of generic fitness and learning apps that attempt to drive engagement through rankings and metrics, there are also more experimental interfaces that employ tangibility to create a bridge between users and their data. For example, the Memstone [5] is a tangible interface to control sharing of personalized video data with others. Physio-Stacks [31] and TOBE [16] follow a similar approach, however, the focus is on sharing personal physiological data with others to support extrospection. Other related interface concepts which focus on exploring one's own data, i.e. introspection, include Teegi [15], a tangible interface showing the user's brainwave data, or the prototypes by Aslan et al. [2], which provide tangible representations of the user's heartbeat and breathing.Similarly, various projects analyze users' engagement with their own fitness data through tangible means, e.g. Activity Sculptures [36] and LOOP [35], which tend to result in an increased amount of self-reflection among users during the prototype deployment.

\subsection{Physical Interfaces for Time Management}

While extensive research exists on the mechanisms and philosophies for personal time management, there appears to be little work using tangible physical interfaces to address task management. Monomizo [19] by Jang et al. follows a hybrid approach by providing a digital calendar widget on the user's desk while also being capable of printing a tangible single-use paper calendar to take along. Similarly, Hojmose presents DailyStack [17] which uses a metaphor of stacking colorful wooden blocks on a platform to represent a stack of tasks to be completed. Ahmed et al. [1] have implemented a tangible approach to time management by extending on the classic marble answering machine by Bishop et al. [6]. The concept is novel and utilises Pomodoro technique to minimise interruptions by embodying characteristics of ambient displays and tangible user interfaces (TUIs). This work has yet to be evaluted in the wild, which motivated us to conduct user studies to learn from user feedback on the perception of physical interfaces for time management. Another notable work with physical interfaces is done by Yuzawa et al. [39], who used a sociotechnical approach through the concept of a Japanese Garden to support multitasking. Their user study of their prototype reported no significance towards productivity, although collaborating partners sent significantly fewer coordination messages, fewer inappropriate messages, and produced fewer interruptions. This directly affects reduced 
stress levels in their users. Another recent tangible prototype, DayClo as developed by Lee et al. [26], presents the advantage of embodying physical interaction onto an everyday object like a table clock. Their longitudinal user study showed that DayClo stimulated users' casual access to their upcoming daily schedules and brought the user's soft alertness, which was not perceived as a pressure but rather as a motivation to self-track to perform their schedules as planned. The tangible interface concepts can be seen in Figure 2.

\subsection{Self Reflection to Develop Routines}

Routines are a series of actions or type of behaviour that are regularly and invariably followed by someone. Moreover, they give a start- and endpoint to a process and thereby provide a feeling of satisfaction. However, people often are in doubt of their productivity in the absence of feedback such as time spent and task engagement [30]. The role of self reflection as a personalised medium to support data interpretation, especially of user generated data, has been described by Ruckstein et al. [33]. In the context of supporting routines, awareness and consciousness of a person is increased when there is a possibility to reflect. This can be seen through the material representation of user generated data like heart beats during an activity into physical artifacts and drinkable souvenirs [20]. People shared experiences of feeling rewarded, pleasantly surprised and a positive indicator to develop a healthy routine (see e.g., [21, 22]). There have also been some contradictions regarding self reflection developed through user generated data as shown by Nim et al. [28]. Their observations reported increased anxiety and stress in people due to the burden of staying consistent with routines everyday. This occurred due to excessive tracking of multiple data features and frequent notifications from smartphone applications. This brings us to the understanding of the importance of integrating self reflection as an ambient form of connection that people can associate with awareness as a positive process.

\subsection{Tangible Rewards}

Rewards are signs of recognition towards the efforts made by a person. HCI research has understood this relevance and studied this perspective in relation to developing routines for people. A recent longitudinal study conducted by Cherubini et al. [10] has shown an unexpected downside when monetary compensation was given to people for collecting step counts. Additionally, motivational notifications were sent to people in the hope of positively nudging them towards collecting more step counts. Both had detrimental effects as the people were unable to associate themselves with such a rewarding system to maintain a routine of walking a certain number of steps everyday. This negative effect was related to the non-personalisation of goals and was not beneficial to trigger motivation for people who are at the initial phase of building exercise routines. Furthermore, the standard predefined goals do not instill a sense of appreciation due to generalising the reward for the people. There are other works that explore personalised reward systems and show the positive effects of tangible rewards. This can be seen through the feature of storing reward by Botros et al. [9] and using it on a day when the person is unable to fulfil their goals which increase consistency. Edipulse by Khot et al. [23] show how the use of surprise personalised messages on 'eatables' creates curiosity and increases motivation for people to exercise regularly.

The above listed research papers show us a spectrum of the pros and cons associated with multimodal interactions for QSW in building routines to people. We will now utilise this information towards developing design criteria for a prototype that can be evaluated in the wild with people which we hope would benefit from efficient task organisation and execution while WFH.

\section{CONCEPT AND PROTOTYPE}

The design of the prototype plays a crucial role in our proposed user studies. Our design goal was for the prototype to enable a sense of focus and awareness in the user's work, thereby supporting people's ability to detach from their work during leisure time. An online survey was conducted with 15 people working at a university, all of whom had their work routines significantly affected by the lockdown measures. The goal of the survey was to understand the data features in which people are interested in when assessing their work day productivity and to observe their preferred modality to set task reminders. Additionally, the survey indicated that respondents continued working both at the university and at their home office while adhering to social distancing rules. The survey described the context of use for the prototype along with a short video explaining the Rainmaker percussion instrument. This ensured that participants were familiar with the form factor and interaction possibilities. The survey contained the following questions:

(1) What is your work environment situation at the moment? (Example: Working only at home or also at the office).

(2) How many tasks do you usually schedule on average? (Example: Noting down some points on sticky notes to be scratched throughout the work day).

(3) Please pick your preferred colour choices in an ascending manner that could represent your work versus break time ratio (three choices could be picked, see Appendix Figure 8).

(4) Imagine you forget to switch the 'Rainmaker' back into 'work'-position but have already continued working. Would you want to receive a reminder either from the smartphone or from the rainmaker itself (e.g., through haptic vibrations or audio)?

(5) What is the amount of time where you can usually work continuously without taking a break? (To learn if there can be an intervention such as vibrations to help people become consistent with work intervals).

(6) What colour do you associate with tasks pending and done? (a choice of red, blue, and green were provided).

Based on the survey responses, we determined three design criteria for the prototype. An illustrative interaction flow with Rainmaker, in which all of the three aforementioned design criteria are represented, is shown in Figure 3.

\section{Metaphorical relation}

Our first criteria was to design a device that offers a physical handle to manipulate a virtual to-do list while taking advantage of multiple sensory channels of human interaction with digital information. The choice of embodying the percussion instrument Rainmaker showed the ideal properties of taking advantage of people's well 


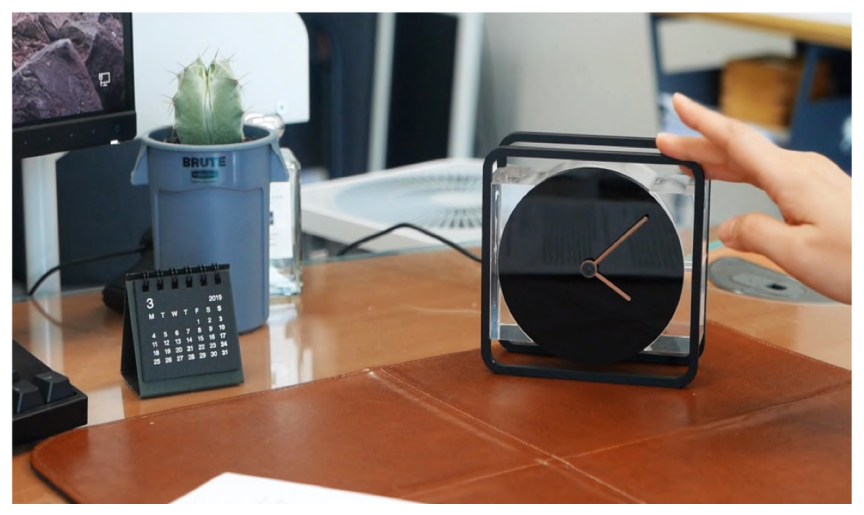

(a) DayClo: Embodying Self Reflection [26]

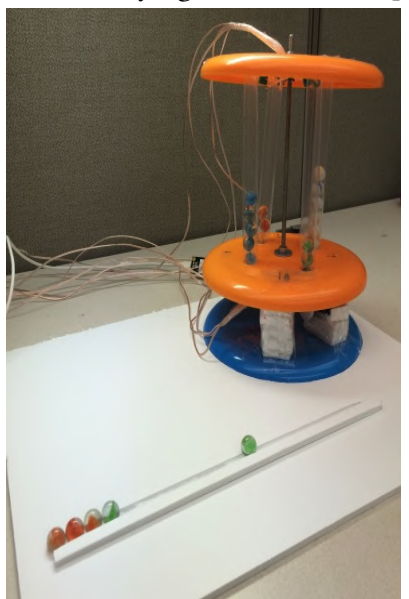

(c) Tangible Pomodoro Timer [1]

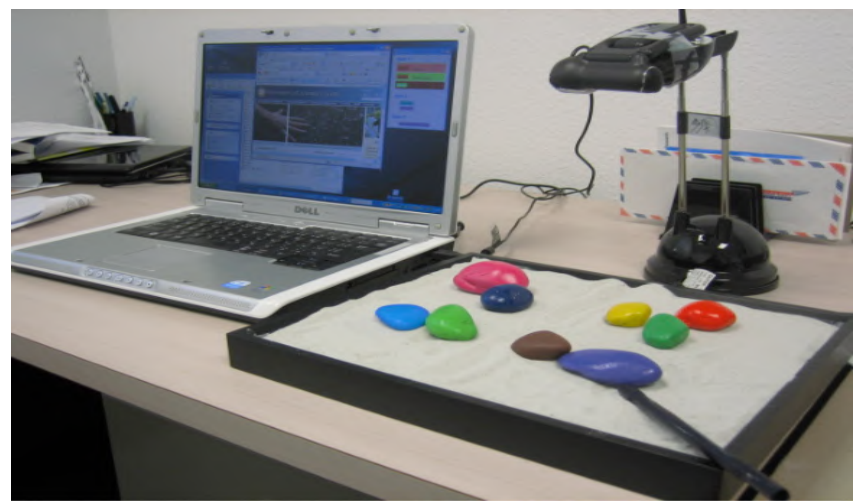

(b) Japanese Garden for Multitasking [39]

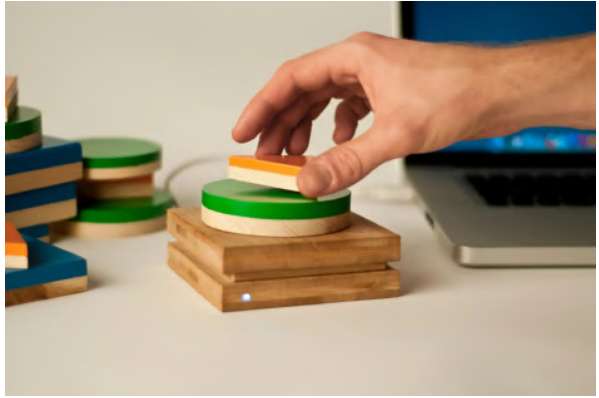

(d) DailyStack [17]

Figure 2: Existing Tangible Time Management Models

entrenched skills and experiences of interacting with non-digital objects; in our case the association with the 'Rainmaker' instrument as suggested by Jacob et al. [18]. Those familiar with the instrument have a strong analogy between the interface and actions in the real world, thereby supporting the metaphor as proposed by Fishkin et al.'s tangible interaction framework [14]. The simulated rainfall sound can also be expected to have a stress-reducing effect, as discussed by Gould van Praag et al. [38]. Green seemed to be the preferred choice for people to associate with their tasks. When further probed, we learnt that green provided them with a positive feeling of accomplishing the tasks. The survey participants seemed positively intrigued by the audio created from the rainfall effect in the survey video, which shows a Rainmaker being played by a musician. The participants suggested the use of this feedback in the context of a work companion prototype (e.g., the rainfall audio giving them a positive indicator of achieved progress). This further supported our choice of embodying a multimodal interaction onto the Rainmaker while validating the findings from Lee et al. [26].

\section{Portability and ease of use}

Second, it is important that people can easily transport the prototype in order to use it at different locations both within their house and outside (e.g., their office). The survey indicated that most of the participants preferred working in their university office space 2-3 times per week, with the remaining days spent working at home. Additionally, ease of use of the prototype was critical in order to avoid distracting users from their actual work or introducing additional barriers to use. We minimized the initial setup process to establishing a Bluetooth connection between a smartphone application and the prototype. Finally, we ensured that the casing was able to protect the electronics in case of incidents in the use of our prototype during the user studies.

\section{Minimising screen interaction}

Third, in contrast to many existing work-routine solutions that are completely digital, our aim was to focus primarily on a physical device. While an extra smartphone application may seem to contradict the purpose of a fully tangible approach for time management, the main intent for the app is to acts as a place to list the task names before beginning to work, creating a starting point 
and bringing awareness of planning the list of tasks. The app also supports firmware updates on the prototype in case of any technical difficulties faced by the participants during the user studies. Additionally, we wanted to keep the option to display the statistics related to time used per task and to have a visualisation of weekly and monthly work times in order for people to track their routines if desired.

\subsection{Implementation}

The outer casing of the prototype was 3D-printed around an existing Rainmaker toy. This toy already contained metal balls that created the audio effect of rainfall when the prototype was physically manipulated. The electrical components consist of a Huzzah ESP 32 micro-controller, an addressable LED strip, 6-axis inertial measurement unit (IMU), and a rechargeable battery. Figure 4 shows an exploded view of the Rainmaker. The LED strip has 10 LEDs in order to support the mean number of tasks of the people we surveyed. The choice of green as blinking (pending task) and solid green (completed task) was also selected as based on the outcomes of the survey. The work versus break time colours on the LED were chosen to be blue for work time and orange for break time, this was done based on the most preferred user choice being option 1 from Figure 8. An Android application was developed to connect to the Rainmaker and manipulate (e.g., add, edit, remove) the tasks. We publicly share the code of the Android application and microcontroller, technical specifications of the electronic components, and the circuit diagram for connecting the electronic components ${ }^{1}$.

A second iteration of the Rainmaker was developed based on the feedback from the first user study. The rechargeable batteries were changed from $350 \mathrm{~mA}$ to $1000 \mathrm{~mA}$ to reduce power discharge during usage of the prototype. We further decided to log usage data of people in the second iteration in order to supplement our interview questions with usage data logs. The communication process can be seen in Figure 5. We furthermore modified the interactions for the second iteration, see Figure 6, as based on the results from the first user study. A Pomodoro timer was added so the LED strip could represent the 25 minutes interval work times and the corresponding interaction of flipping the prototype to activate the timer resonates with the cylindrical form factor providing the user a feeling of handling a physical hour glass.

The interactions mentioned in Figure 3 and 6 are detected by the micro-controller using the output from the (IMU), specifically the Y-axis readings. For this purpose, the rotation angle on the Y-axis is constantly being stored and checked. A turn-over event is triggered if the change in rotation is above a certain threshold in one direction and under a limited period of time. The same applies for the shake event, however, the threshold here is smaller and has to be reached 3 times in both directions alternately. These conditions also have to be met under a limited period of time. The time limit and the threshold for the rotation change are important to avoid false positive detection, i.e. unintentional turning over/shaking.

\section{QUALITATIVE USER STUDIES}

While our primary research goal of exploring the effective use of multimodal interactions drove the design and implementation

\footnotetext{
${ }^{1}$ https://github.com/mmbuw/RainMaker.git
}

of Rainmaker, we wanted go further at evaluating our choice of features through a qualitative user study. Referring to Boehner et al.'s discussion of probes in HCI [8], our approach can be considered a 'technology probe', i.e. an exploratory study and not so much a usability study of a nearly-finished product. We conducted two separate user studies on two iterations of the prototype, using semistructured interviews after the participants had spent time using the prototype. The user studies were guided by the research questions below.

- RQ 1: In the context of working from home, what is the role of multimodal interactions in triggering a positive effect towards productivity in accomplishing tasks?

- RQ 2: Does intermediate reflection on the utilized time with a ratio of working versus break time increase the awareness of the working hours?

- RQ 3: Does the integration of the Pomodoro timer technique in the form of a tangible interactive medium encourage its use for a more focused time management along with the tasks organization feature in the prototype?

RQ 1 and RQ $\mathbf{2}$ were explored during Study 1, whereas RQ 3 was explored in Study 2 along with additional feedback to answer RQ 1. Table 1 shows the differences between the two user studies that were conducted on the prototype. RQ $\mathbf{1}$ and $\mathbf{R Q} \mathbf{2}$ were generated at the start of the Study 1 and eventually $\mathbf{R Q} 3$ was generated for Study 2 based on the feedback we received from Study 1 . The primary motivation to conduct Study 2 was to learn more about the influence of TUIs on work routines as the time spent by participants in Study 1 allowed us to mainly understand about the pragmatic and hedonic effects of the Rainmaker, but we were unable to establish relations between the participants and their work routine. Therefore, we focused on a longer study duration in Study 2 with a lower number of participants.

\section{STUDY 1}

In Study 1, participants were encouraged to integrate the use of the Rainmaker into their regular work day to allow us to learn from their interactions and perceptions developed in the time spent with the Rainmaker over the course of the user studies. We encouraged participants to keep their normal schedule of either working from home or their office space, and participants could carry the prototype with them if needed.

\subsection{Participants}

We recruited 11 participants for Study 1. Eight participants are researchers at a university, one participant is a student currently working on his thesis, and two participants are engineers working for a company. All the participants were actively working from their homes or isolated in their personal offices during the course of the user study. The participants were expected by their employers to work 8 hours everyday from Monday to Friday while the student self-managed his work hours with no fixed limit. The median age of the participants was 27.5 years.

\subsection{Procedure and Analysis}

An explanation on the background of creating the prototype along with a demonstration of the functionalities of the Rainmaker in 


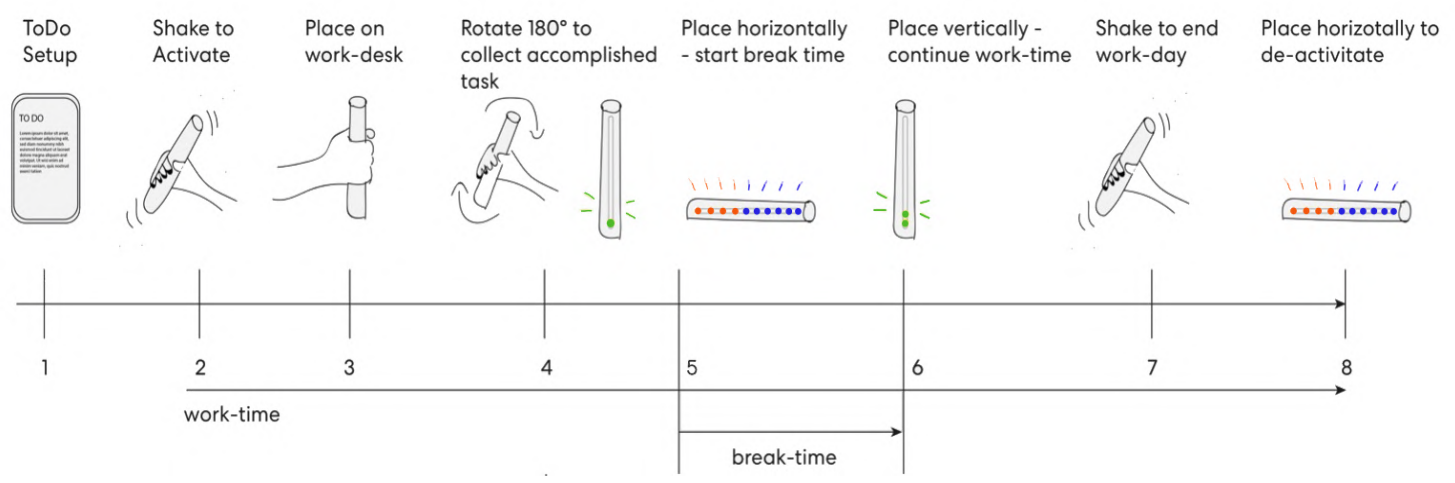

Figure 3: Rainmaker interactions for Study 1.

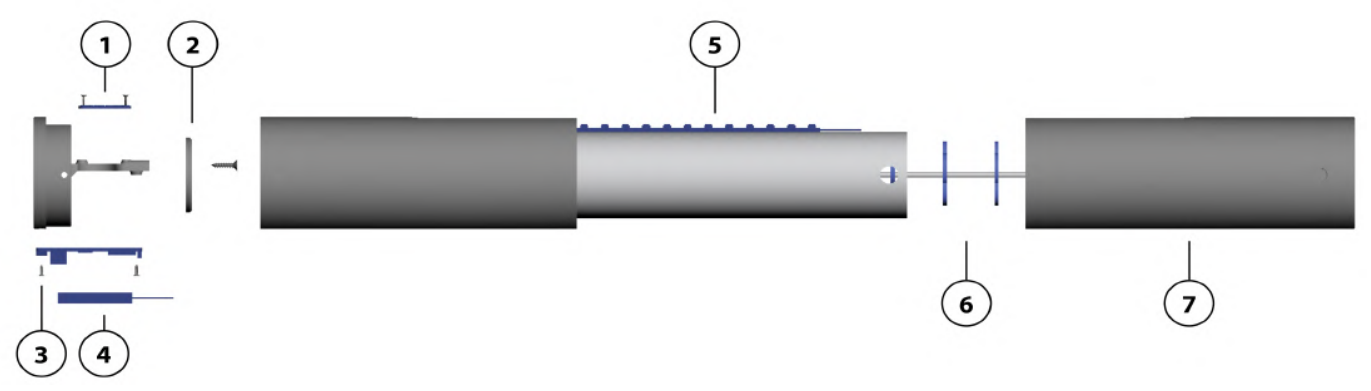

Figure 4: Components of the Rainmaker. 1) Inertial measurement unit, 2) Electronic components locking cap, 3 ) ESP32, 4) Battery, 5) LED, 6) Layers to control flow of the acoustic balls, 7) Outer Casing.

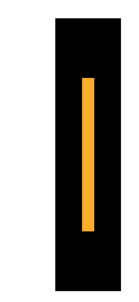

RainMaker

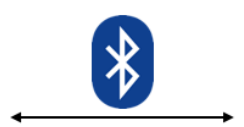

Android Application
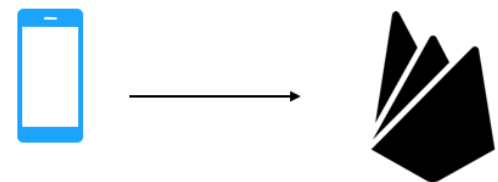

- Task Title

- Time Ratio

- Frequency of Use

Firebase Analytics

Figure 5: Rainmaker Communication Cycle.

coordination with the Android app was provided to participants at the start of the study. A manual, see Appendix Figure 9, with all available interactions was also handed out to familiarise our participants with the prototype during the first use. Participants were met again after three days for a semi-structured feedback interview. The minutes of the interview were audio recorded during the course of the conversation after taking the consent from the participants. We probed on the effects of the form factor, the choice of metaphor and interactions, the information perceptions, and the overall gratification of the users.

\subsection{Results Study 1}

The below emergent themes are influenced by the questions from the semi-structured interview conducted with the eleven participants of Study 1 at the end of their three days of using the prototype.

5.3.1 Form Factor and Material. The size and width of the Rainmaker was felt indifferent by most participants. Two participants stated while in the horizontal position, they had to make space for the Rainmaker to be placed on their work table. One participant stated that when there are more than seven tasks, little fatigue was felt in the forearm while flipping the rainmaker for every task completed. Four participants would prefer to have the Rainmaker in a wood casing for aesthetic reasons. 


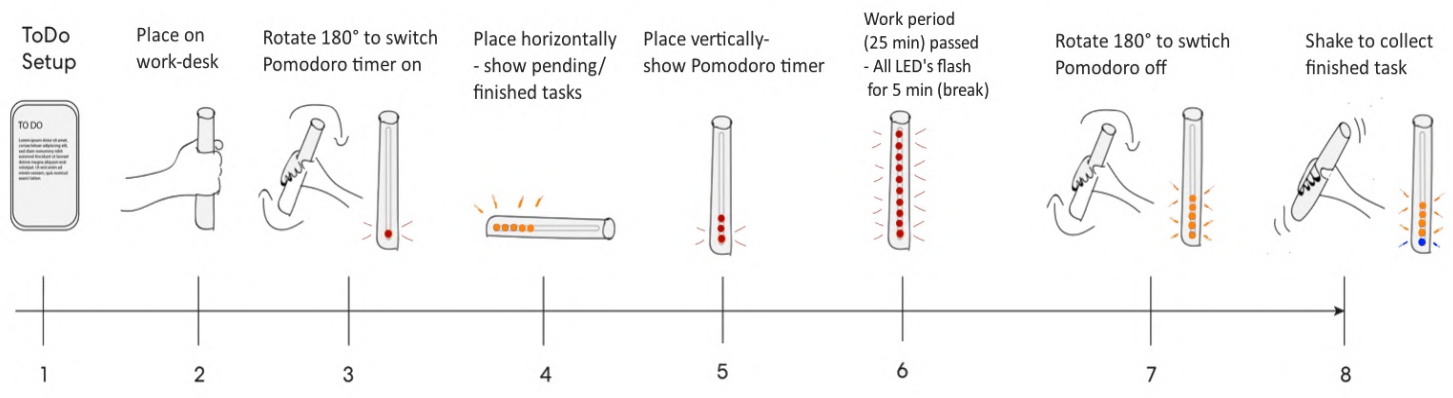

Figure 6: Rainmaker interactions for Study 2.

\begin{tabular}{lll}
\hline Parameter & Study $\mathbf{1}$ & Study $\mathbf{2}$ \\
\hline No. of participants & 11 & 2 \\
Study duration & 3 days & 15 days \\
Context & Isolated office space & Isolated office space \\
Data logging & No & Yes \\
Feedback & Recorded interviews & Recorded interviews \\
Data Features & Number of Tasks, Work vs Break time & Number of Tasks, Pomodoro Timer \\
Reflection Trigger & Ratio of time & Ratio of tasks \\
Pending Tasks & Blinking Green & Orange \\
Completed Tasks & Solid Green & Blue \\
Time Feedback & Work vs Break time & Time passed on Pomodoro Timer \\
Interactions & See Figure 3 & See Figure 6 \\
\hline
\end{tabular}

Table 1: Overview of our two user studies.

5.3.2 Metaphor. The positions of vertical and horizontal symbolizing work and break were understood immediately by the participants. Seven participants explicitly stated that they liked this idea of positions reflecting work and break mode. Two participants mentioned that the vertical position of the Rainmaker on the table was visualised as their coworkers sitting across and working. This motivated them to reduce their procrastination and continue working towards the end of their work day. One participant stated "the routine of starting my work by shaking the rainmaker gets me motivated to tick off the tasks scheduled for the day".

5.3.3 Visual Effects and Acoustics. The LED lights received positive feedback towards immediate satisfaction in synchronisation with the reward of the rainfall effect. All participants from Study 1 were looking forward to this interaction during their work day which made them aware of the tasks to be completed. However, one participant stated "The LEDs are too bright when facing me directly as they strain my eyes and especially when I work in the night without background lights, the LEDs further strain my eyes. It would be cooler to have an option to dim the LEDs or put them off when needed". This prompted us to look into changing the intensity and colours of the LED lights for future iterations. Most participants appreciated the sound of the rainfall effect, while were two participants who felt the rainfall effect could be shortened or varied for different tasks. One participant stated, "I enjoy the sound when my work environment has been silent for sometime, the rainfall sound brings me joy that one task is out of my way and I could mostly take a break. I could relate this (the rainfall sound) to moving on with my day".

5.3.4 Reflection mode. The work vs break time ratio represented intermediate and final feedback when placed horizontally. While participants appreciated this feature during the demonstration, it was later learnt from their feedback that most forgot to place the Rainmaker horizontally to start the break time. This misrepresented the final ratio that was to be reflected. The process of starting the work day by turning the Rainmaker on and adding tasks through the smartphone provided a preparation phase for participants to decide their tasks for the day. Some participants would prefer the Rainmaker rewarding them with break times while progressing on a huge task similar to the Pomodoro concept so they can still receive some reward in between large tasks when they are unable to flip the Rainmaker more frequently. The Rainmaker provided a sense of work organisation and one participant also said "The Rainmaker works well to break bad habits of procrastinating that affect my work." Similar thoughts were echoed by other participants about making them more conscious on their pending tasks through the physical presence of the Rainmaker. One participant stated "The work vs break time is useless to me as I am not interested in reflecting 
this as it brings a negative feeling when the break time shown is significant while I actually worked as expected. And, when I want to take a break like go have coffee, this is more important for me than remembering to place the Rainmaker horizontal to measure my break time". Further, participants noted that it was not always easy to distinguish work from breaks "it was difficult to categorise work and break on few occasions when I multitask mundane tasks with eating snacks". This feedback was valuable in redefining reflection for the second iteration.

Figure 7a shows the Rainmaker being used by a Study 1 participant for learning about the cumulative work time versus break time made so far in the day. The Rainmaker helped in increasing the awareness of the tasks, which were organised as sub-tasks on each sticky note and as a single main task on the rainmaker. Figure $7 \mathrm{~b}$ shows a participant using the task mode of the Rainmaker to refer to what is completed and pending by placing it on a side table close to the working table.

\section{STUDY 2}

Study 2 followed the same overall structure as Study 1, but ran over a longer period of time and with a lower number of participants. The feedback from participants of study 1 were considered and the Pomodoro feature was added to the Rainmaker as a result and RQ 3 was subsequently added to the objectives of the overall user study.

\subsection{Participants}

Study 2 was conducted with two participants. The first participant was a part-time student who also worked part-time at an architectural company. She is currently working on her master thesis. Her work environment is her home office for both company-related work and her thesis. The second participant worked at a university as a full time researcher. She mostly worked from her home and also worked at her personal office space at the university at least once per week.

\subsection{Procedure and Analysis}

In Study 2, we once again handed out the prototype along with the Android application. A manual was not necessary as this was not found very useful from Study 1 as we observed the participants learning the functionalities immediately during the demonstration. We conducted three semi-structured interviews during the course of the 15 day study - an initial interview to understand their current state of work routines, an intermediate interview to learn their experience after a week with the prototype, and finally a feedback interview to seek their views and to understand the effect of the device on their productivity.

\subsection{Results Study 2}

The following emergent themes have been gathered from the semistructured interviews conducted at different phases of Study 2 with the two participants. The participant with an architectural background will be referred to as $\mathrm{P} 1$ and the participant with a research background will be referred to as $\mathrm{P} 2$ in this section.

6.3.1 Type of Tasks. We observed from the log files that both participants used the prototype for a similar type of task. Upon analysis, it could be observed that these tasks were mundane, repetitive, and reminders. Some examples from the $\mathrm{P} 1$ were Sketch floor plans, Complete layout drawing, Finish electric grid sketches, and Complete the references for the document. P2 also had similar types of tasks namely Upload presentation, Write a mail to Mr. X, Finish Paper, Start proposal, and Ask Mrs. Y.

It can be seen that the task names of both participants showed similarities in the adjectives used to describe the task. This was later confirmed by the participants that the tasks were repetitive, but important to be ticked off for their day's work. P1 stated that "It was a very good way to organise my boring tasks right at the start of the work day. The rainmaker helped me do this because it was the first thing I saw on my work table". P2 stated that "The Rainmaker was really useful to me for getting my tasks that I am not looking forward to out of my way as soon as possible".

6.3.2 Pomodoro Timer. The addition of this feature was influenced from the participant feedback we received in Study 1. We also felt the cylindrical form factor of the prototype would be analogous to an hourglass which would make the interaction of flipping the prototype natural to turn on/off the Pomodoro timer.

The use of Pomodoro timer was less than expected from both P1 and P2. Interestingly, P1 used the Pomodoro timer that was meant for 25 minutes of work interval as a break countdown timer when she was doing her time-intensive creative tasks. She stated "I flipped the Rainmaker mostly for my lunch breaks as I felt the 25 minutes is a good break to have lunch and get back to work. I liked the reference of a countdown timer". P2 felt the Pomodoro timer worked well for her mostly towards the end of the work day when she was fatigued and still had pending tasks. She stated "I usually turned the Pomodoro on after $4 p m$ when I have the final emails to be sent out. By having a countdown timer, I would try to focus and get the work done".

6.3.3 Visual Effects and Acoustics. This is the backbone feature of our design and we wanted to strengthen our understanding from the participants of Study 2 through their longer engagement with the prototype. As mentioned earlier, the visual effects were modified to a more eye-friendly colour scheme showing pending tasks in orange and completed tasks in blue. Both participants showed positive contention towards the lights in correlation to tasks. P1 stated "The lights directly affected my work environment and it was always my intention to see the orange lights turn blue as I felt satisfied when this happened". P2 backed this by stating "The lights gave me a sense of progress to my boring tasks and having a physical feedback for this gave me a good experience and also some motivation to get done with them (tasks)".

The acoustics of the rainfall effect was the byproduct of completing tasks and should provide a feeling of reward for this action. Both participants had opinions about the tangible reward of the acoustics. P1 stated that "I enjoyed shaking it (rainmaker) whenever I finished the tasks. I generally like sounds and this effect was good for me. I was hoping if we could also add sub tasks on the mobile so I could shake it (rainmaker) more often to feel good". While P2 also appreciated this acoustic reward, her excitement was less than P1's. P2 stated "I liked the rainfall sound in general but when I flip it (rainmaker) the duration of the sound felt longer. Maybe I would prefer a shorter and softer rainfall effect". 


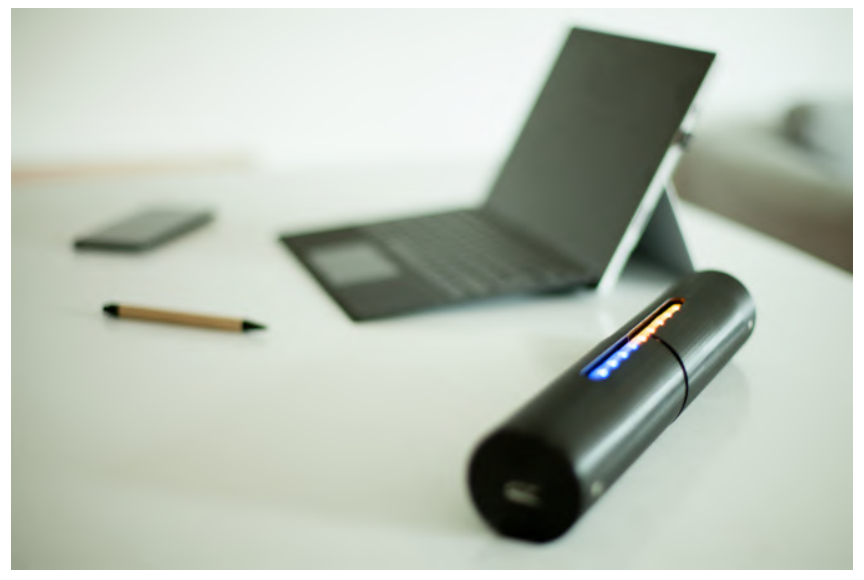

(a) Rainmaker placed for intermediate reflection.

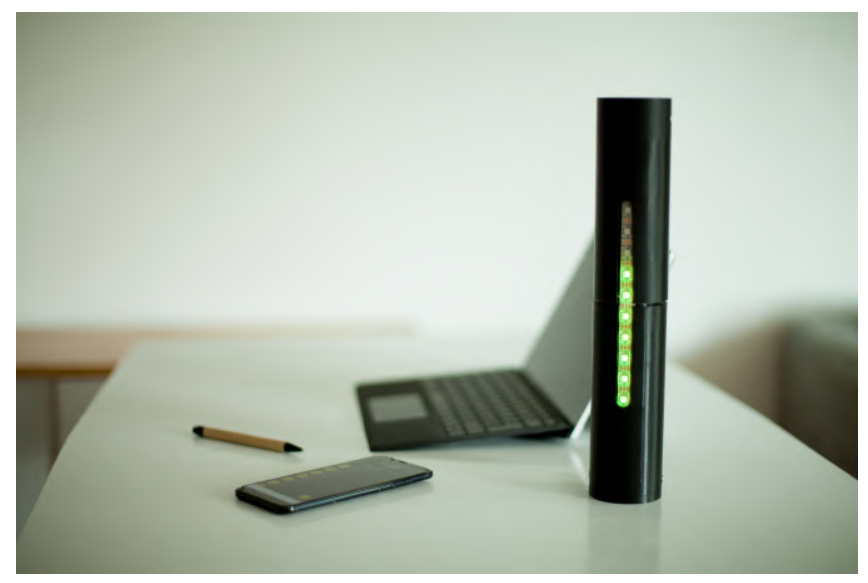

(b) Rainmaker used for task reference.

Figure 7: Rainmaker in the work environment of participants during study 1.

6.3.4 Participant Reflection. Self reflection is vital while working as it allows us to bring awareness of how we are conducting our work. We wanted to stimulate this effect through the prototype. The intermediate reflection from the first prototype iteration of work vs break time was removed. In this user study, we wanted our participants to share their reflective moments from their time of engagement with the prototype.

Both participants had their own reflective moments while using the Rainmaker, there were similarities in their experience which showed awareness, sense of progress and excitement to finish tasks. P1 stated "I enjoy this routine of doing something with my hands when I finish tasks. It is a satisfaction for me". P2 stated "I like the presence of it(Rainmaker) on my table as it motivates me to prepare the boring tasks to be done and I manage to do this better now(after having the rainmaker) than before".

6.3.5 Work Routines. We were able to observe the start and end times of work days from logging the time when the first task was added to the time when all the tasks were done. The routines of P1 could not be established as consistent. Her start times were mostly in the morning but the range of start times was 3 hours. Furthermore, P1 also had start time recorded later in the day and also in late evenings. This correlates to her background of managing her own work hours depending on the workload. P2 recorded more stable start and end times. She mostly started her work sessions with the Rainmaker late mornings with a range of 1 hour among all the recorded start times. She only started 1 session at noon. All her sessions have ended by early evening. This correlates to her work hours from mornings to early evenings.

\section{DISCUSSION}

In the previous sections, we listed distinct emergent themes from both user studies. We will describe our interpretations from both user studies separately and converge towards the common insights as obtained throughout this project.

\subsection{Study 1}

The participants shared a resounding yes to integrating the Rainmaker into their work environment. The engagement of the multimodal interactions that utilised touch, vision, and sound was well received by the participants. Some participants felt that the Rainmaker motivated them to break habits of being unproductive. The work vs break time as a reflection mode did not produce the desired effect we had hoped for. We speculate this is due to the interaction of placing the Rainmaker horizontally to activate the break time which some participants forgot, as this interaction is the only different spatial position compared to other vertical positions.

On the other hand, the interaction of completing tasks was well received due to the rainfall effect. From this observation, the lack of acoustic effect for the break mode may have contributed to participants forgetting about the reflection feature. However, from the interviews we learnt that participants felt more immediate satisfaction with taking the break instead of interacting with the Rainmaker. We also observed that participants did not associate measuring break times as a strong factor to define their productivity. Most participants suggested they would benefit more from seeing time spent for each task, allowing them to reflect better on their work day.

In these unprecedented times, home office seems to continue for an indefinite time. However, people would still want to collaborate for work. We tried to explore this direction of a multi-user scenario, as participants suggested collaboration features such as the LED strip showing a common task with two or more people and colour coding to reflect the sub-task designation between the team members. This could motivate them to finish the tasks sooner and the tangibility would make the experience more enjoyable while being physically isolated. In contrast, several participants felt the Rainmaker is best kept as a personal reflection tool since a team mode could become overwhelming and might encourage unnecessary competition. 


\subsection{Study 2}

We observed further effects of the prototype with the participants and it could be seen that a certain type of tasks were well suited for organisation and execution through the Rainmaker. These tasks were described as mundane and laborious. Both participants previously kept tab on these tasks mentally, but the prototype in their work environment provided them with a reference to completed and pending tasks. They added these tasks at the start of the work day on the prototype when they were fresh as compared to without the prototype. This routine increased their chances of being aware of the boring tasks while they were engrossed in other work tasks. They felt more successfully in completing these mundane tasks with the help of the Rainmaker.

While the use of Pomodoro timer was less than anticipated, the timer was effective for the participants nevertheless. Both participants used the timer towards the end of the work day, mostly when there were one or two pending tasks remaining. The countdown timer acted as a positive indicator that they needed to work for a few more minutes to complete the task on hand. The aspect of countdown timer was further appreciated by P1 when she used the Pomodoro timer for her lunch breaks, since the countdown timer gave her a good indication of time left in the break.

The LED lights directly stimulated the work environment of the participants, as the physical presence of the light allowed participants to reflect whether it was the right time to procrastinate from work. Participants positively used the influence of the lights to carry on with work when their mind felt like wandering away from the tasks on hand. P1 suggested that when she sat on her bed unexpectedly in the middle of work, the awareness to go back and work was heightened upon on seeing orange lights (pending tasks). The rewarding effect from the acoustics was subjective for both $\mathrm{P} 1$ and $\mathrm{P} 2$ but inclined towards a positive effect. This indicates the importance of creating a tangible reward suited for an individual, as mentioned earlier in Section 2.4.

Study 2 was conducted on an iteration of the prototype influenced by the feedback from Study 1 so the choice of interactions were modified. Both participants were comfortable with the features and associated interactions. The shake interaction to tick off completed task was well appreciated by both participants. They also preferred to do this action more often and hoped to have sub tasks listed on the prototype that could be ticked off through a similar shake physical interaction.

The information gathered for the start and end times of the work sessions recorded through the prototype resonated their background of working style. It could be observed that both participants added more tasks when they used the Rainmaker in the mornings before 10:00. There were instances of more than one session with the prototype recorded on the same day, usually after noon. This happened when the participants were able to finish their tasks listed in the mornings. This behaviour in the participants was influenced due the effect of the physical feedback of the mundane tasks from the prototype. The tangible presence of the pending tasks made them aware and this directly nudged them to complete these tasks. Furthermore, both participants appreciated this positive change brought through the Rainmaker about increasing their chances of completing the added tasks that resulted in a satisfactory work day.

\subsection{Takeaways \& Future Work}

It can be seen from our interpretations of both user studies that participants were able to associate the interactions with the metaphorical relation to the percussion instrument - Rainmaker. The shorter Study 1 provided us valuable feedback on what worked well and and what did not work so well for the participants in the context of work from home. The important learning we took forward from Study 1 was that the multimodal interaction, in our case rainfall effect (acoustics) and lights (visual), was well received. We furthermore noticed that participants experienced satisfaction of ticking off tasks through a physical action with their hands. We utilised this learning in Study 2 with a multimodal interaction to trigger a Pomodoro timer to provide our users with a way to stay focused with their time. This feature was mostly used by participants towards the end of the day when their energy levels were low. The effect of the countdown timer from the LED and its interaction that created acoustics brought in a sense of motivation to get the tasks done for the day. Additionally, it could be seen that participants in Study 2 associated strongly to contrasting colours of pending tasks (orange) and completed tasks (blue) compared to the single-coloured green light from Study 1. This shows the strong information association with people when contrasting effect is used for its representation. In Study 1, we learnt that the prototype promotes breaking habits that negatively affect work but it could be seen in more detail from Study 2 that participants increased their chances to finish mundane tasks earlier in the day leading to satisfaction in creating time for creative tasks. This change was influenced through the use of Rainmaker that provided a tangible reference for pending and completed tasks.

From our observations and interpretations developed through the two user studies, it can be inferred that certain characteristics have to be personalised for every participant. This is evident through the unanimous inclination for the prototype to be a personal awareness device while working. The characteristics can be seen as follows:

- Complexity: This is associated with the interactions and the corresponding data representation. While one can argue that the data representation is a linear mapping of tasks added through the phone but the tangibility of using hands to tick off a completed task was effective to the participants. Such a routine will keep the participants engaged with using the prototype on a regular basis in supporting their work routines. Similar aspects of introducing an ideal level of complexity with data and interaction on other data features like time spent on tasks can be investigated to provide an overview of habits related to work for the participants over varying periods of time. The choice of colours played a pivotal role in creating awareness, in Study 1 the use of only green had a lesser impact as compared to the use of two distinctive colours in Study 2 for task status. This observation also corresponds to the use of distinctive colours for tasks in [39].

- Tangible Rewards: This remains a highly subjective characteristic to such a prototype as evident from the results of the user studies. The range of highly appreciated to no appreciation towards the acoustic effect was seen amongst the 
participants. This was further subjective with their alternate desires of haptics, other acoustic effects, and also having varied level of sound depending on the time spent per task. Therefore, we would consider this to be a personal choice that can be incorporated for every individual's preference for a successful effect, e.g. by modifying the amount of acoustic balls inside the device. This characteristic further supports the aspects of adding more variations such as storing rewards and creating curiosity to the reward system based on an individual's preference, as seen in $[9,23]$.

- Reflection: We observed the importance of reflection to be influenced through the data that affects the work day This was found to be the ratio of pending versus completed tasks. The routine of using hands to change the ratio of pending and completed tasks directly created a sense of satisfaction towards progress in the work day. This was more clear from our observations in Study 2 when P1 felt the satisfaction of blue lights increasing and the corresponding shake interaction that caused the acoustic effect. Therefore, introduction of reflection is most effective through a tangible change that defines the quality of the work day.The process of engaging users with multimodal interactions brings about increased awareness and consciousness to support reflective moments which can be seen here through the visual and acoustic effects of ticking of the tasks. This prototype also conforms with other self reflection prototypes such as [25, 34] that bring about awareness through reflection in the contexts of mental well being and parent-child interactions respectively.

\section{CONCLUSION}

In this paper we presented the development and evaluation of Rainmaker, a physical prototype intended to support in work routines in the context of work from home. We evaluated our prototype in two user studies ( $N=11$ for three days each, and $N=2$ for 15 days each) to obtain insights into participants' perceptions of and engagement with Rainmaker. We found that multimodal interactions resulted in a positive effect on task awareness, in particular for tasks that were considered mundane and time intensive. Participants made use of the prototype to develop work routines through which they were able to more effectively finish their more mundane tasks and subsequently free time and mental space for more engaging tasks and leisure time. Through Study 1, we learnt that participants reflected primarily on their task ratio (pending versus completed tasks) as opposed to the ratio of work versus break time - resulting in a negative perception of the work day - and that our participants required more concrete support to manage their time. Through the iteration made for Study 2, reflection among participants increased due to a more meaningful visualisation of pending versus completed tasks and the ability to make use of a Pomodoro timer. Although appreciated, the timer functionality was primarily used when participants felt fatigued towards the end of the day. Interestingly, the visual effect of the countdown timer of Pomodoro was highly appreciated by both participants of Study 2 and could be used in other scenarios, e.g. for longer breaks such as lunch. Our study highlights the challenges experienced by the forced WFH and the opportunity for novel interventions to support users in their daily professional life.

\section{ACKNOWLEDGMENTS}

This work was funded by the Deutsche Forschungsgemeinschaft (DFG, German Research Foundation) through project EC437/1-1. We thank product designers Leoni Fischer and Pauline Temme who are former students at Bauhaus Universität, Germany for their contributions with the prototype illustrations, material and design suggestions during the initial phase of the project. Finally, we thank our participants in supporting our work with their feedback during the user studies.

\section{REFERENCES}

[1] Ryan Ahmed, Alex Chambers, Michael Frontz, and Stephen Voida. 2014. A tangible approach to time management. In Proceedings of the 2014 ACM International Joint Conference on Pervasive and Ubiquitous Computing: Adjunct Publication. 207-210.

[2] Ilhan Aslan, Hadrian Burkhardt, Julian Kraus, and Elisabeth André. 2016. Hold My Heart and Breathe with Me: Tangible Somaesthetic Designs. In Proceedings of the 9th Nordic Conference on Human-Computer Interaction (Gothenburg, Sweden) (NordiCHI '16). Association for Computing Machinery, New York, NY, USA, Article 92, 6 pages. https://doi.org/10.1145/2971485.2996727

[3] Jose Maria Barrero, Nicholas Bloom, and Steven J Davis. 2020. Why Working From Home Will Stick. University of Chicago, Becker Friedman Institute for Economics Working Paper 2020-174 (2020).

[4] Lutz Bellmann and Olaf Hübler. 2020. Working from home, job satisfaction and work-life balance-robust or heterogeneous links? International fournal of Manpower (2020).

[5] Agon Bexheti, Anton Fedosov, Ivan Elhart, and Marc Langheinrich. 2018. Memstone: A Tangible Interface for Controlling Capture and Sharing of Personal Memories. In Proceedings of the 20th International Conference on Human-Computer Interaction with Mobile Devices and Services (Barcelona, Spain) (MobileHCI '18). Association for Computing Machinery, New York, NY, USA, Article 20, 13 pages. https://doi.org/10.1145/3229434.3229477

[6] Durell Bishop. 1992. Marble answering machine. Royal College of Art, Interaction Design (1992).

[7] A. E. Blandford and T. R. G. Green. 2001. Group and Individual Time Management Tools: What You Get is Not What You Need. Personal Ubiquitous Comput. 5, 4 (Jan. 2001), 213-230. https://doi.org/10.1007/PL00000020

[8] Kirsten Boehner, Janet Vertesi, Phoebe Sengers, and Paul Dourish. 2007. How HCI Interprets the Probes. In Proceedings of the SIGCHI Conference on Human Factors in Computing Systems (San Jose, California, USA) (CHI '07). Association for Computing Machinery, New York, NY, USA, 1077-1086. https://doi.org/10. 1145/1240624.1240789

[9] Fadi Botros, Charles Perin, Bon Adriel Aseniero, and Sheelagh Carpendale. 2016. Go and grow: Mapping personal data to a living plant. In Proceedings of the International Working Conference on Advanced Visual Interfaces. 112-119.

[10] Mauro Cherubini, Gabriela Villalobos-Zuñiga, Marc-Olivier Boldi, and Riccardo Bonazzi. 2020. The Unexpected Downside of Paying or Sending Messages to People to Make Them Walk: Comparing Tangible Rewards and Motivational Messages to Improve Physical Activity. ACM Transactions on Computer-Human Interaction (TOCHI) 27, 2 (2020), 1-44.

[11] Prithwiraj Choudhury, Cirrus Foroughi, and Barbara Zepp Larson. 2020. Workfrom-anywhere: The productivity effects of geographic flexibility. In Academy of Management Proceedings, Vol. 2020. Academy of Management Briarcliff Manor, NY 10510, 21199

[12] Mihaly Csikszentmihalyi. 2000. Beyond boredom and anxiety. Jossey-Bass.

[13] Antonio Ferreira, Luca Bertolini, and Petter Næss. 2017. Immotility as resilience? A key consideration for transport policy and research. Applied Mobilities 2, 1 (2017), 16-31.

[14] Kenneth P Fishkin. 2004. A taxonomy for and analysis of tangible interfaces. Personal and Ubiquitous computing 8, 5 (2004), 347-358.

[15] Jérémy Frey, Renaud Gervais, Stéphanie Fleck, Fabien Lotte, and Martin Hachet. 2014. Teegi: Tangible EEG Interface. In Proceedings of the 27th Annual ACM Symposium on User Interface Software and Technology (Honolulu, Hawaii, USA) (UIST '14). Association for Computing Machinery, New York, NY, USA, 301-308. https://doi.org/10.1145/2642918.2647368

[16] Renaud Gervais, Jérémy Frey, Alexis Gay, Fabien Lotte, and Martin Hachet. 2016. TOBE: Tangible Out-of-Body Experience. In Proceedings of the TEI '16: Tenth International Conference on Tangible, Embedded, and Embodied Interaction 
(TEI '16). Association for Computing Machinery, New York, NY, USA, 227-235 https://doi.org/10.1145/2839462.2839486

[17] Anders Hojmose. 2010. Daily Stack. https://www.hojmo.se/work/daily-stack/.

[18] Robert JK Jacob, Audrey Girouard, Leanne M Hirshfield, Michael S Horn, Orit Shaer, Erin Treacy Solovey, and Jamie Zigelbaum. 2008. Reality-based interaction: a framework for post-WIMP interfaces. In Proceedings of the SIGCHI conference on Human factors in computing systems. 201-210.

[19] Sangsu Jang, Subin Kim, Boram Noh, and Young-Woo Park. 2019. Monomizo: A Tangible Desktop Artifact Providing Schedules from E-Ink Screen to Paper. In Proceedings of the 2019 on Designing Interactive Systems Conference (San Diego, CA, USA) (DIS '19). Association for Computing Machinery, New York, NY, USA 1123-1130. https://doi.org/10.1145/3322276.3322333

[20] Rohit Ashok Khot. 2016. Understanding material representations of physical activity. Ph.D. Dissertation. RMIT University.

[21] Rohit Ashok Khot, Jeewon Lee, Deepti Aggarwal, Larissa Hjorth, and Florian'Floyd' Mueller. 2015. Tastybeats: Designing palatable representations of physical activity. In Proceedings of the 33rd Annual ACM Conference on Human Factors in Computing Systems. 2933-2942.

[22] Rohit Ashok Khot and Florian" Floyd" Mueller. 2013. Sweat-atoms: turning physical exercise into physical objects. In CHI'13 Extended Abstracts on Human Factors in Computing Systems. 3075-3078.

[23] Rohit Ashok Khot, Ryan Pennings, and Florian 'Floyd' Mueller. 2015. EdiPulse: supporting physical activity with chocolate printed messages. In Proceedings of the 33rd Annual ACM Conference Extended Abstracts on Human Factors in Computing Systems. 1391-1396.

[24] Young-Ho Kim, Jae Ho Jeon, Eun Kyoung Choe, Bongshin Lee, KwonHyun Kim, and Jinwook Seo. 2016. TimeAware: Leveraging Framing Effects to Enhance Personal Productivity. Association for Computing Machinery, New York, NY, USA, 272-283. https://doi.org/10.1145/2858036.2858428

[25] Kwangyoung Lee and Hwajung Hong. 2017. Designing for self-tracking of emotion and experience with tangible modality. In Proceedings of the 2017 Conference on Designing Interactive Systems. 465-475.

[26] Kyung-Ryong Lee, Somi Ju, Temirlan Dzhoroev, Geonil Goh, Moon-Hwan Lee, and Young-Woo Park. 2020. DayClo: An Everyday Table Clock Providing Interaction with Personal Schedule Data for Self-reflection. In Proceedings of the 2020 ACM Designing Interactive Systems Conference. 1793-1806.

[27] Phoebe V Moore. 2017. The quantified self in precarity: Work, technology and what counts. Routledge.

[28] Evgeniya Nim. 2019. Digital Self-Tracking Among Russian Students: Practices And Discourses. Higher School of Economics Research Paper No. WP BRP 91 (2019).

[29] Tom Owen, Jennifer Pearson, Harold Thimbleby, and George Buchanan. 2015 ConCap: Designing to Empower Individual Reflection on Chronic Conditions Using Mobile Apps. In Proceedings of the 17th International Conference on HumanComputer Interaction with Mobile Devices and Services (Copenhagen, Denmark) (MobileHCI '15). Association for Computing Machinery, New York, NY, USA 105-114. https://doi.org/10.1145/2785830.2785881

[30] Leslie A Perlow. 1999. The time famine: Toward a sociology of work time Administrative science quarterly 44, 1 (1999), 57-81.

[31] Joan Sol Roo, Renaud Gervais, Thibault Lainé, Pierre-Antoine Cinquin, Martin Hachet, and Jérémy Frey. 2020. Physio-Stacks: Supporting Communication with Ourselves and Others via Tangible, Modular Physiological Devices. In 22nd International Conference on Human-Computer Interaction with Mobile Devices and Services (Oldenburg, Germany) (MobileHCI '20). Association for Computing Machinery, New York, NY, USA, Article 19, 12 pages. https://doi.org/10.1145/ 3379503.3403562

[32] Ori Rubin, Anna Nikolaeva, Samuel Nello-Deakin, and Marco te Brömmelstroet 2020. What can we learn from the COVID-19 pandemic about how people experience working from home and commuting? Centre for Urban Studies, University of Amsterdam (2020).

[33] Minna Ruckenstein and Natasha Dow Schüll. 2017. The datafication of health Annual Review of Anthropology 46 (2017), 261-278.

[34] Ofir Sadka, Hadas Erel, Andrey Grishko, and Oren Zuckerman. 2018. Tangible interaction in parent-child collaboration: encouraging awareness and reflection. In Proceedings of the 17th ACM Conference on Interaction Design and Children. 157-169.

[35] Kim Sauvé, Saskia Bakker, Nicolai Marquardt, and Steven Houben. 2020. LOOP Exploring Physicalization of Activity Tracking Data. In Proceedings of the 11th Nordic Conference on Human-Computer Interaction: Shaping Experiences, Shaping Society (Tallinn, Estonia) (NordiCHI '20). Association for Computing Machinery, New York, NY, USA, Article 52, 12 pages. https://doi.org/10.1145/3419249.3420109

[36] S. Stusak, A. Tabard, F. Sauka, R. A. Khot, and A. Butz. 2014. Activity Sculptures: Exploring the Impact of Physical Visualizations on Running Activity. IEEE Transactions on Visualization and Computer Graphics 20, 12 (2014), 2201-2210. https://doi.org/10.1109/TVCG.2014.2352953

[37] Niels van Berkel, Chu Luo, Denzil Ferreira, Jorge Goncalves, and Vassilis Kostakos. 2015. The Curse of Quantified-Self: An Endless Quest for Answers. In Adjunct Proceedings of the 2015 ACM International foint Conference on Pervasive and Ubiquitous Computing and Proceedings of the 2015 ACM International Symposium on Wearable Computers (Osaka, Japan) (UbiComp/ISWC'15 Adjunct). Association for Computing Machinery, New York, NY, USA, 973-978. https://doi.org/10. $1145 / 2800835.2800946$

[38] Cassandra D. Gould van Praag S. Garfinkel, Oliver Sparasci, Alex Mees, Andrew O. Philippides, M. Ware, C. Ottaviani, and H. Critchley. 2017. Mind-wandering and alterations to default mode network connectivity when listening to naturalistic versus artificial sounds. Scientific Reports 7 (2017). https://doi.org/10.1038/ srep45273

[39] Hideto Yuzawa and Gloria Mark. 2010. The Japanese garden: task awareness for collaborative multitasking. In Proceedings of the 16th ACM International Conference on Supporting Group Work. 253-262.

\section{A SUPPORTING ILLUSTRATIONS}

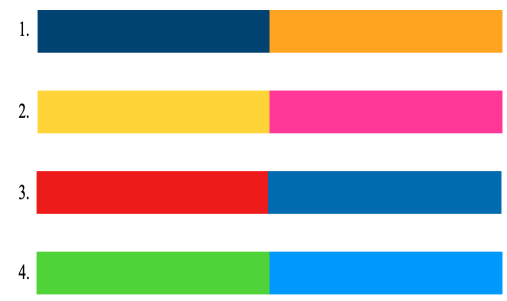

Figure 8: 'Work' versus 'Break time' colour choices 


\section{Rainmaker User Manual}

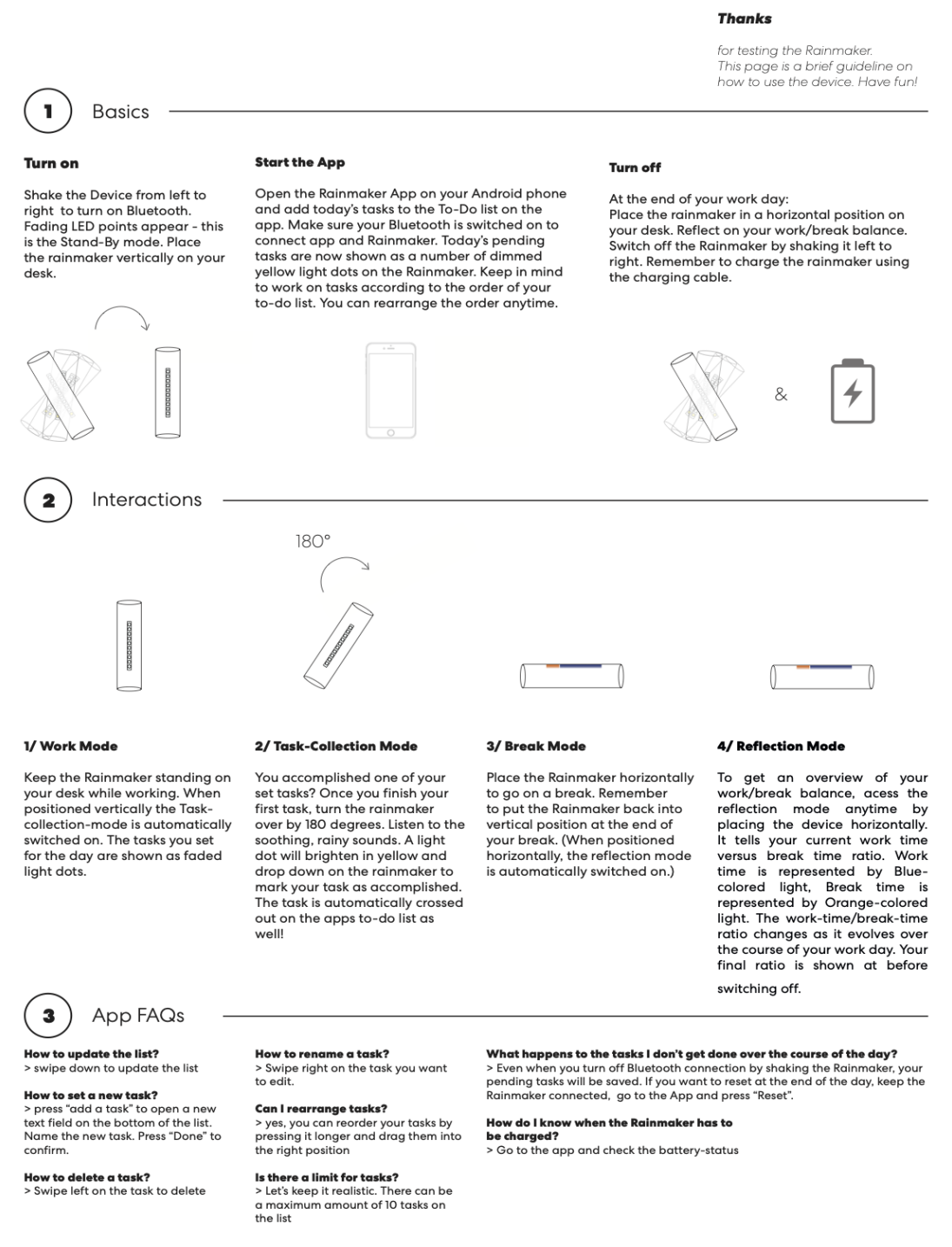

Figure 9: Study 1 User Manual 